\title{
Prices Versus Quantities : Stock Pollution Control with Repeated Choice of the Instrument
}

M. Germain and A. Magnus

Discussion Paper 2005-48

\section{Département des Sciences Économiques de l'Université catholique de Louvain}




\title{
Prices versus quantities: stock pollution control with repeated choice of the instrument
}

\author{
by \\ Marc Germain ${ }^{2}$ \\ and \\ Alphonse Magnus ${ }^{3}$
}

September 2005

\begin{abstract}
One examines strategies of pollution control through choices between taxes and tradable permits, supposed to be decided at several time periods $t_{1}, t_{2}, \ldots, t_{I}$. At each of these time periods, the choice depends on the solution of a dynamic programming problem involving the expectations of the polluting factor of production function $z(t)$ and the pollutant stock function $S(t)$. According to coefficients of these two functions in the dynamic problem, it is shown under a broad functional setting that permits are decided for a while, followed by decisions of taxes for all the remaining periods. Finite $(I<\infty)$, as well as infinite $(I=\infty)$ horizon is considered.
\end{abstract}

\footnotetext{
${ }^{1}$ This research is part of the CLIMNEG2 project and the Belgian Programme on Interuniversity Attraction Poles, both initiated by the Belgian Science Policy. The scientific responsibility rests with its authors. The authors wish to thank Raouf Boucekkine and Vincent van Steenberghe for stimulating discussions and suggestions.

${ }^{2}$ Département d'Economie and CORE, Université Catholique de Louvain (Email : germain@core.ucl.ac.be).

${ }^{3}$ Institut de Mathématique, Université Catholique de Louvain (Email : magnus@anma.ucl.ac.be).
} 


\section{Introduction}

Taxes and quotas of tradable pollution permits are two important and intensively studied economic instruments for pollution control. These paper is concerned with the comparison of taxes and permits when the regulatory authority and the polluters have asymmetric information about abatement costs, and when environmental damages are due to a pollutant that accumulates. At each period of time, the regulator chooses the type and the level of the instrument that maximises a welfare functional depending on the expected flow of production minus damage costs, taking in account the expected answer of the polluters to the chosen environmental policy.

We depart from the literature concerned by this subject (a.o. Hoel and Karp [1,2], Newell and Pizer [3], Pizer [4]) by allowing the possibility of switching between instruments at each period, by considering finite as well as infinite horizon frameworks, and by considering a more general formulation of the regulator's objective and of the pollutant accumulation.

We do not restrict ourselves to the infinite horizon case studied in the above mentioned literature where the choice of the instrument appears to be constant through time, and we show in the finite horizon case that permits are decided for a while, followed by decisions of taxes for all the remaining periods.

\section{The model}

The model has both continuous and discrete time features. $t$ is the continuous time variable $(0 \leq t \leq H)$, which is divided in periods of length $h$, which are indexed by the discrete time index $i(i \in\{1,2, \ldots, I\}$, with $h=H / I)$. The regulator chooses the instrument and its level at the beginning of each period $i$, and keeps this level constant for the whole period.

To this end, one must estimate the reaction of the polluting firms:

\subsection{The polluting firm reaction.}

At each step $i$, the representative polluting firm is assumed to maximise a quadratic profit functional involving

$$
a_{i} z(t)-\frac{b}{2} z^{2}(t)-\tau_{i} z(t), \quad[i-1] h \leqslant t \leqslant i h,
$$

in which $\left[a-\frac{b}{2} z\right] z$ is the firm's production function, where $z$ is the polluting factor of production (for example, emissions linked to energy), $a_{i}$ is the realization at period $i$ of $a$, a discrete i.i.d. random process with mean $\mu$ and variance $\sigma^{2}$, and $b$ is a positive constant. $\tau_{i}$ is either the level of the tax chosen by the regulator, or the observed price of permits at period $i$. In problem (1), it is assumed that the representative firm does not take account of the future, because it is too small to influence the aggregate stock of pollutant, and thus has no power to influence the future environmental policy. The firm is thus unable to manipulate the future decisions of the regulator, so that its profit maximisation problem is solved at any time by

$$
z(t)=z_{i}:=\frac{a_{i}-\tau_{i}}{b},[i-1] h \leq t \leq i h
$$

If the chosen instrument at step $i$ is a tax, the behaviour of the firm is described by (2). If the chosen instrument are permits, then whatever the random shock $a_{i}$, their price will adjust so that the demand of permits by polluters equals the quantity of permits supplied by the regulator, $\bar{x}_{i}$. In that case,

$$
z(t)=\bar{x}_{i},[i-1] h \leq t \leq i h
$$




\subsection{The stock of pollutant.}

We suppose that the evolution of the stock of pollutant $S(t)$ is given in $t \in[(i-1) h, i h]$ by

$$
S(t)=\alpha(t-[i-1] h) S_{i-1}+\beta(t-[i-1] h) z_{i},
$$

where $S_{i-1}$ is the value $S([i-1] h)$, and $z_{i}$ is the value seen above of the piecewise constant function $z(t)$ on $([i-1] h, i h) . \alpha$ and $\beta$ are continuous functions, and $\alpha(0)=1, \beta(0)=0$.

A typical instance of (4) results when $S(t)$ is a solution of the differential equation

$$
\frac{d S(t)}{d t}=z(t)-\delta S(t), S(0)=S_{0} \text { given }
$$

where $\delta$ is the rate of decay of the stock of pollution $(\delta>0)$ and where the number of firms has been normalized to 1 . As $z(t)$ is the constant $z_{i}$ on $([i-1] h, i h)$, we solve immediately (5) as

$$
S(t)=\frac{z_{i}}{\delta}+\left[S_{i-1}-\frac{z_{i}}{\delta}\right] \exp (-\delta(t-[i-1] h)),
$$

whence

$$
\alpha(t)=\exp (-\delta t), \quad \beta(t)=\frac{1-\exp (-\delta t)}{\delta} .
$$

\subsection{The regulator's decision.}

The stock of pollutant $S(t)$ results in damages to society that are equal to $\pi S^{2}(t) / 2$, where $\pi$ is a positive parameter.

Let $\xi$ be a binary decision variable determining the type of the instrument (tax or permits), with $\xi=0$ if permits are chosen and $\xi=1$ if a tax is chosen. Let $x=E\{z\}$ be the expectation of $z$. Given (2) and (3), $x$ can be taken as the decision variable determining the level of the instrument (the level of the tax or the quantity of permits), whether this instrument is a tax or a quota.

At the beginning of each step $i$ (i.e. when $t=[i-1] h$ ), that is before observing the current and future random shocks $a_{j}(j=i, \ldots, I)$, but knowing however their mean and variance, the regulator chooses the type and the level of the instrument that maximise the expected flow of payoffs defined as the difference between production and damages $\pi S^{2} / 2$, subject to the behaviour of the polluters (described by (2) or (3)) and to the stock equation (5). At step $i$, the regulator solves the following dynamic programming problem :

$$
V_{i}\left(S_{i-1}\right)=\max _{\xi_{i}, x_{i}} E\left\{\mathcal{F}_{i}\left(a(t) z(t)-\frac{b}{2} z^{2}(t)-\frac{\pi}{2} S^{2}(t)\right)\right\},
$$

subject to (4) and, given (2) and (3), to

$$
z(t)=x_{i}+\xi_{i}\left[\frac{a_{i}-\mu}{b}\right]
$$

with $V_{I+1}\left(S_{H}\right)=0$ and where $S_{j}:=S(j h)$, and where we only require $\mathcal{F}_{i}$ to be a linear positive functional involving functions on $[i-1] h<t<H$, and submitted to the condition (10) below.

A typical example of what we have in mind is an integral

$$
\mathcal{F}_{i}(f)=\int_{[i-1] h}^{H} f(t) \exp (-r(t-[i-1] h)) d t
$$

where $r$ is the exogeneous (positive) discount rate, but other forms have been considered (see examples later on). 
We also suppose that the functionals $\mathcal{F}_{i}$ and $\mathcal{F}_{i+1}$ are related by

$$
\mathcal{F}_{i}(f)=\mathcal{F}(f(t+[i-1] h))+\varepsilon \mathcal{F}_{i+1}(f),
$$

where $\mathcal{F}$ involves functions defined on $t \in[0, h]$.

Remark that $\mathcal{F}_{i}(f)=\sum_{j=i}^{I} \varepsilon^{j-i} \mathcal{F}(f(t+[j-1] h))$.

In our example $(9), \mathcal{F}(f)=\int_{0}^{h} f(t) e^{-r t} d t$, and $\varepsilon=e^{-r h}$. We then have $\mathcal{F}(1)=\frac{1-e^{-r h}}{r}$, from $(6), \mathcal{F}(\alpha)=\frac{1-e^{-[r+\delta] h}}{r+\delta}, \mathcal{F}(\beta)=\frac{1-e^{-r h}}{r \delta}-\frac{1-e^{-[r+\delta] h}}{[r+\delta] \delta}$, etc. Using such expressions in the subsequent discussions should soon be an absolute nightmare. That's why we shall keep the $\mathcal{F}$ notation in further calculations as far as possible. Our theory is therefore able to cope with several formulas. As a second example, Hoel and Karp use in [1] an elementary integration formula $\mathcal{F}(f)=\frac{1-e^{-r h}}{r} f(0)$, therefore, their $\mathcal{F}_{i}(f)$ is $\frac{1-e^{-r h}}{r} \sum_{j=i}^{I} e^{-r[j-i] h} f([j-1] h)$.

\section{Optimal solution.}

Theorem. For any set of functionals $\mathcal{F}_{i}$ satisfying (10), the problems (7) are solved with values $\xi_{i}=0$ (permits) or $\xi_{i}=1(\operatorname{tax})$ determined whether the ratio $\pi / b$ is larger or smaller than a computable value $\eta_{i}$. Moreover, $\eta_{1}<\eta_{2}<\cdots<\eta_{I}$.

If $H \rightarrow \infty$ (infinite horizon), $\eta_{1}, \eta_{2}, \ldots$ tend towards a same limit $\eta^{*}$.

This means that,

(1) if $\pi / b<\eta_{1}$, taxes will be chosen for all periods,

(2) if $\pi / b>\eta_{I}$, quotas will be chosen for all periods,

(3) if $\eta_{1}<\pi / b<\eta_{I}$, quotas will be chosen while $\pi / b>\eta_{i}$, and taxes later on.

When the horizon is infinite, we have taxes or permits for all the periods, depending only on the ratio $\pi / b$, whether it is smaller or larger than $\eta^{*}$.

\section{Proof.}

(1) We first look at how the decision has to be taken with respect to the last period $i=I$. We perform the calculation of (7), using (4), and knowing that $a$ and $x$ (and therefore $z$ ) are constants on $\left(t_{I-1}, t_{I}\right)=(H-h, H)$ :

$$
\begin{gathered}
\mathcal{F}_{I}\left(a_{I} z_{I}-\frac{b}{2} z_{I}^{2}-\frac{\pi}{2}\left[\alpha(t-[I-1] h) S_{I-1}+\beta(t-[I-1] h) z_{I}\right]^{2}\right) \\
=\mathcal{F}\left(a_{I} z_{I}-\frac{b}{2} z_{I}^{2}-\frac{\pi}{2}\left[\alpha(t) S_{I-1}+\beta(t) z_{I}\right]^{2}\right) \quad \text { from (10) } \\
=\left(a_{I} z_{I}-\frac{b}{2} z_{I}^{2}\right) \mathcal{F}(1)-\frac{\pi}{2}\left[S_{I-1}^{2} \mathcal{F}\left(\alpha^{2}\right)+2 z_{I} S_{I-1} \mathcal{F}(\alpha \beta)+z_{I}^{2} \mathcal{F}\left(\beta^{2}\right)\right]
\end{gathered}
$$

which shows already that the result will be a quadratic polynomial in $S_{I-1}$.

We now compute the mathematical expectation in (7), knowing that

(a) when $\xi_{I}=1(\operatorname{tax}), a_{I}$ is a value of a random variable of mean $\mu$ and variance $\sigma^{2}$. Therefore, from (8), $E\left\{z_{I}\right\}=x_{I}, E\left\{a_{I} z_{I}\right\}=E\left\{a_{I} x_{I}+\left(a_{I}^{2}-\mu a_{I}\right) / b\right\}=\mu x_{I}+\sigma^{2} / b$, 
and $E\left\{z_{I}^{2}\right\}=E\left\{x_{I}^{2}+2 x_{I}\left(a_{I}-\mu\right) / b+\left(a_{I}-\mu\right)^{2} / b^{2}\right\}=x_{I}^{2}+\sigma^{2} / b^{2}$. So,

$$
\begin{gathered}
E\left\{\mathcal{F}_{I}\left(a_{I} z_{I}-\frac{b}{2} z_{I}^{2}-\frac{\pi}{2} S^{2}(t)\right)\right\} \\
=\left(\mu x_{I}+\frac{\sigma^{2}}{b}-\frac{b}{2} x_{I}^{2}-\frac{b}{2} \frac{\sigma^{2}}{b^{2}}\right) \mathcal{F}(1) \\
\quad-\frac{\pi}{2}\left[S_{I-1}^{2} \mathcal{F}\left(\alpha^{2}\right)+2 x_{I} S_{I-1} \mathcal{F}(\alpha \beta)+\left[x_{I}^{2}+\frac{\sigma^{2}}{b^{2}}\right] \mathcal{F}\left(\beta^{2}\right)\right] \\
=\frac{\sigma^{2}}{2 b} \mathcal{F}(1)-\frac{\pi}{2} S_{I-1}^{2} \mathcal{F}\left(\alpha^{2}\right)-\frac{\pi}{2} \frac{\sigma^{2}}{b^{2}} \mathcal{F}\left(\beta^{2}\right)+\left[\mu \mathcal{F}(1)-\pi S_{I-1} \mathcal{F}(\alpha \beta)\right] x_{I}-\left[b \mathcal{F}(1)+\pi \mathcal{F}\left(\beta^{2}\right)\right] \frac{x_{I}^{2}}{2}
\end{gathered}
$$

There is only one variable left, which is $x_{I}$. Maximizing with respect to this variable, i.e., by putting

$$
x_{I}=\frac{\mu \mathcal{F}(1)-\pi S_{I-1} \mathcal{F}(\alpha \beta)}{b \mathcal{F}(1)+\pi \mathcal{F}\left(\beta^{2}\right)},
$$

one finds the required quadratic polynomial in $S_{I-1}$

$$
\frac{\sigma^{2}}{2 b}\left[\mathcal{F}(1)-\frac{\pi}{b} \mathcal{F}\left(\beta^{2}\right)\right]-\frac{\pi}{2} S_{I-1}^{2} \mathcal{F}\left(\alpha^{2}\right)+\frac{\left[\mu \mathcal{F}(1)-\pi S_{I-1} \mathcal{F}(\alpha \beta)\right]^{2}}{2 b\left[\mathcal{F}(1)+\frac{\pi}{b} \mathcal{F}\left(\beta^{2}\right)\right]} .
$$

(b) When $\xi_{I}=0$ (permits), from (8), $z_{I}=x_{I}$, so that $E\left\{z_{I}\right\}=x_{I}, E\left\{a_{I} z_{I}\right\}=\mu x_{I}$, and $E\left\{z_{I}^{2}\right\}=x_{I}^{2}$. Now

$$
\begin{aligned}
E\left\{\mathcal { F } _ { I } \left(a_{I} z_{I}-\frac{b}{2} z_{I}^{2}-\frac{\pi}{2}\right.\right. & \left.\left.S^{2}(t)\right)\right\}=\left(\mu x_{I}-\frac{b}{2} x_{I}^{2}\right) \mathcal{F}(1) \\
& -\frac{\pi}{2}\left[S_{I-1}^{2} \mathcal{F}\left(\alpha^{2}\right)+2 x_{I} S_{I-1} \mathcal{F}(\alpha \beta)+x_{I}^{2} \mathcal{F}\left(\beta^{2}\right)\right] \\
& =-\frac{\pi}{2} S_{I-1}^{2} \mathcal{F}\left(\alpha^{2}\right)+\left[\mu \mathcal{F}(1)-\pi S_{I-1} \mathcal{F}(\alpha \beta)\right] x_{I}-\left[b \mathcal{F}(1)+\pi \mathcal{F}\left(\beta^{2}\right)\right] \frac{x_{I}^{2}}{2}
\end{aligned}
$$

This is maximized at the same value of $x_{I}$ as before. The mathematical expectation is therefore the same as (12), but without the $\sigma^{2}$ factor.

One shall therefore choose $\xi_{I}=1(\operatorname{tax})$ if this latter factor is positive, and $\xi_{I}=0$ otherwise.

The complete solution of ( 7$)$ at the final period is

$$
V_{I}\left(S_{I-1}\right)=-\frac{\pi}{2} S_{I-1}^{2} \mathcal{F}\left(\alpha^{2}\right)+\frac{\left[\mu \mathcal{F}(1)-\pi S_{I-1} \mathcal{F}(\alpha \beta)\right]^{2}}{2 b\left[\mathcal{F}(1)+\frac{\pi}{b} \mathcal{F}\left(\beta^{2}\right)\right]}+\xi_{I} \frac{\sigma^{2}}{2 b}\left[\mathcal{F}(1)-\frac{\pi}{b} \mathcal{F}\left(\beta^{2}\right)\right]
$$

We emphasize the quadratic character of the function $V_{I}$ by writing (13) as

$$
V_{I}\left(S_{I-1}\right)=\rho_{0}^{(I)}+\rho_{1}^{(I)} S_{I-1}+\frac{\rho_{2}^{(I)} \pi}{2} S_{I-1}^{2},
$$

(borrowing a notation of [1]).

Remark that $\rho_{2}^{(I)}=\pi \frac{\mathcal{F}^{2}(\alpha \beta)-\mathcal{F}\left(\alpha^{2}\right) \mathcal{F}\left(\beta^{2}\right)-[b / \pi] \mathcal{F}(1) \mathcal{F}\left(\alpha^{2}\right)}{b \mathcal{F}(1)+\pi \mathcal{F}\left(\beta^{2}\right)}$ is negative: as $\mathcal{F}$ is a positive linear functional, $\mathcal{F}(\alpha \beta)$ is a scalar product.

The sign of the coefficient of $\xi_{I}$ in (13) tells if tax or permit will be decided for the last period, i.e., whether

$$
\eta_{I}=\frac{\mathcal{F}(1)}{\mathcal{F}\left(\beta^{2}\right)} \lessgtr \frac{\pi}{b}
$$


In the simple case where $\mathcal{F}(f)=f(h)$ and $\beta(t)=t$, the result is $1 / h^{2}$. If $\mathcal{F}(f)=\int_{0}^{h} f(t) d t$, $\eta_{I}=3 / h^{2}$. And if $\mathcal{F}(f)=f(0), \eta_{I}=\infty$ (always tax during the last period).

(2) We come now to the general problem (7) for an intermediate period $([i-1] h, i h)$.

We will show by induction that a quadratic expression similar to (14), i.e.,

$$
V_{i+1}\left(S_{i}\right)=\rho_{0}^{(i+1)}+\rho_{1}^{(i+1)} S_{i}+\frac{\rho_{2}^{(i+1)} \pi}{2} S_{i}^{2},
$$

holds for all $i$. This is important because, using (10), and the solution of (7) at $i+1$,

$\mathcal{F}_{i}\left(a(t) z(t)-\frac{b}{2} z^{2}(t)-\frac{\pi}{2} S^{2}(t)\right)=\mathcal{F}\left(a_{i} z_{i}-\frac{b}{2} z_{i}^{2}-\frac{\pi}{2} S^{2}(t+[i-1] h)\right)+\varepsilon V_{i+1}\left(S_{i}\right)$,

as all the parameters have been settled in $\mathcal{F}_{i+1}$.

Assuming (16) (which is true when $i=I-1$, from(14)), we proceed as above in looking for the maximum of $E\left\{\mathcal{F}_{i}\right\}$.

(a) If a tax is to be decided at step $i$,

$$
\begin{gathered}
E\left\{\mathcal{F}_{i}\right\}=\left(\frac{\sigma^{2}}{2 b}+\mu x_{i}-\frac{b}{2} x_{i}^{2}\right) \mathcal{F}(1)-\frac{\pi}{2}\left[S_{i-1}^{2} \mathcal{F}\left(\alpha^{2}\right)+2 x_{i} S_{i-1} \mathcal{F}(\alpha \beta)+\frac{\sigma^{2}}{b^{2}} \mathcal{F}\left(\beta^{2}\right)+x_{i}^{2} \mathcal{F}\left(\beta^{2}\right)\right] \\
+\varepsilon \rho_{0}^{(i+1)}+\varepsilon \rho_{1}^{(i+1)}\left[\alpha(h) S_{i-1}+\beta(h) x_{i}\right]+\frac{\varepsilon \rho_{2}^{(i+1)} \pi}{2}\left[\left[\alpha(h) S_{i-1}+\beta(h) x_{i}\right]^{2}+\beta^{2}(h) \frac{\sigma^{2}}{b^{2}}\right] \\
=\frac{\sigma^{2}}{2 b} \mathcal{F}(1)-\frac{\pi}{2} S_{i-1}^{2} \mathcal{F}\left(\alpha^{2}\right)-\frac{\pi}{2} \frac{\sigma^{2}}{b^{2}} \mathcal{F}\left(\beta^{2}\right) \\
+\varepsilon \rho_{0}^{(i+1)}+\varepsilon \rho_{1}^{(i+1)} \alpha(h) S_{i-1}+\varepsilon \frac{\rho_{2}^{(i+1)}}{2} \pi \alpha^{2}(h) S_{i-1}^{2}+\varepsilon \frac{\rho_{2}^{(i+1)} \pi \beta^{2}(h) \sigma^{2}}{2 b^{2}} \\
+\left[\mu \mathcal{F}(1)-\pi S_{i-1} \mathcal{F}(\alpha \beta)+\varepsilon \rho_{1}^{(i+1)} \beta(h)+\varepsilon \rho_{2}^{(i+1)} \pi \alpha(h) \beta(h) S_{i-1}\right] x_{i}-\left[b \mathcal{F}(1)+\pi \mathcal{F}\left(\beta^{2}\right)-\varepsilon \rho_{2}^{(i+1)} \pi \beta^{2}(h)\right] x_{i}^{2} / 2,
\end{gathered}
$$

Maximum is reached at

$$
x_{i}=\frac{\mu \mathcal{F}(1)-\pi S_{i-1} \mathcal{F}(\alpha \beta)+\varepsilon \rho_{1}^{(i+1)} \beta(h)+\varepsilon \rho_{2}^{(i+1)} \pi \alpha(h) \beta(h) S_{i-1}}{b \mathcal{F}(1)+\pi \mathcal{F}\left(\beta^{2}\right)-\varepsilon \rho_{2}^{(i+1)} \pi \beta^{2}(h)},
$$

resulting in

$$
\begin{aligned}
\frac{\sigma^{2}}{2 b} \mathcal{F}(1)-\frac{\pi}{2} S_{i-1}^{2} \mathcal{F}\left(\alpha^{2}\right)-\frac{\pi}{2} & \frac{\sigma^{2}}{b^{2}} \mathcal{F}\left(\beta^{2}\right) \\
+\varepsilon \rho_{0}^{(i+1)}+\varepsilon \rho_{1}^{(i+1)} \alpha(h) S_{i-1}+\varepsilon \frac{\rho_{2}^{(i+1)}}{2} \pi \alpha^{2}(h) S_{i-1}^{2}+\varepsilon \frac{\rho_{2}^{(i+1)} \pi \beta^{2}(h) \sigma^{2}}{2 b^{2}} & \\
& +\frac{\left[\mu \mathcal{F}(1)-\pi S_{i-1} \mathcal{F}(\alpha \beta)+\varepsilon \rho_{1}^{(i+1)} \beta(h)+\varepsilon \rho_{2}^{(i+1)} \pi \alpha(h) \beta(h) S_{i-1}\right]^{2}}{2\left[b \mathcal{F}(1)+\pi \mathcal{F}\left(\beta^{2}\right)-\varepsilon \rho_{2}^{(i+1)} \pi \beta^{2}(h)\right]},
\end{aligned}
$$

(b) If permits are to be decided at step $i$, we perform the same calculations. The maximum is reached at the same value as (17), and the result is the same as above, but without the terms containing $\sigma^{2}$.

We confirm therefore the inductive step from (16)

$$
V_{i}\left(S_{i-1}\right)=\rho_{0}^{(i)}+\rho_{1}^{(i)} S_{i-1}+\frac{\rho_{2}^{(i)} \pi}{2} S_{i-1}^{2},
$$


where we only look at the $\xi_{i} \sigma^{2}$ terms in $\rho_{0}^{(i)}=\xi_{i} \frac{\sigma^{2}}{2 b} R_{i}(\pi / b)+\cdots$ :

$$
R_{i}\left(\frac{\pi}{b}\right)=\mathcal{F}(1)-\frac{\pi}{b} \mathcal{F}\left(\beta^{2}\right)+\varepsilon \frac{\pi}{b} \rho_{2}^{(i+1)} \beta^{2}(h),
$$

so that we also will have to care for $\rho_{2}^{(i)}$ :

$$
\rho_{2}^{(i)}=-\mathcal{F}\left(\alpha^{2}\right)+\varepsilon \rho_{2}^{(i+1)} \alpha^{2}(h)+\pi \frac{\left[-\mathcal{F}(\alpha \beta)+\varepsilon \rho_{2}^{(i+1)} \alpha(h) \beta(h)\right]^{2}}{b \mathcal{F}(1)+\pi \mathcal{F}\left(\beta^{2}\right)-\varepsilon \rho_{2}^{(i+1)} \pi \beta^{2}(h)},
$$

which shows that the $\rho_{2}$ 's, and therefore the $R_{i}$ 's in (19), are rational functions of $\pi / b$.

(3) We proceed now with a close inspection of (20) in order to discuss the positive root $\eta_{i}$ of $R_{i}(\pi / b)=0$, so to determine the value of the ratio $\pi / b$ which will trigger tax or permits during the $i^{\text {th }}$ step:

(a) We work (20), showing that $\rho_{2}^{(i)}$ is actually a rational function of first degree of $\rho_{2}^{(i+1)}$ :

$$
\rho_{2}^{(i)}=\frac{\frac{\pi}{b}\left[\varepsilon \rho_{2}^{i+1} \mathcal{F}\left(\gamma^{2}\right)+\mathcal{F}^{2}(\alpha \beta)-\mathcal{F}\left(\alpha^{2}\right) \mathcal{F}\left(\beta^{2}\right)\right]+\varepsilon \alpha^{2}(h) \mathcal{F}(1) \rho_{2}^{(i+1)}-\mathcal{F}(1) \mathcal{F}\left(\alpha^{2}\right)}{\mathcal{F}(1)+\frac{\pi}{b}\left[\mathcal{F}\left(\beta^{2}\right)-\varepsilon \beta^{2}(h) \rho_{2}^{(i+1)}\right]}
$$

where the function $\gamma$ is $\gamma(t):=\beta(h) \alpha(t)-\alpha(h) \beta(t)$. We already see that all the $\rho_{2}^{(i) \text {, } s}$ are negative when $i \leqslant I$, as $\rho_{2}^{(I+1)}=0$, and as the numerator of (21) is negative $\left(\mathcal{F}^{2}(\alpha \beta) \leqslant \mathcal{F}\left(\alpha^{2}\right) \mathcal{F}\left(\beta^{2}\right)\right.$, and $\left.\mathcal{F}(1) \mathcal{F}\left(\alpha^{2}\right)>0\right)$.

(b) We now proceed with the simple fraction expansion of (21):

$$
\rho_{2}^{(i)}=-\frac{\mathcal{F}\left(\gamma^{2}\right)}{\beta^{2}(h)}-\frac{b}{\pi} \frac{\alpha^{2}(h)}{\beta^{2}(h)} \mathcal{F}(1)+\frac{b}{\pi \beta^{2}(h)} \frac{\left[\alpha(h) \mathcal{F}(1)-\frac{\pi}{b} \mathcal{F}(\beta \gamma)\right]^{2}}{\mathcal{F}(1)+\frac{\pi}{b}\left[\mathcal{F}\left(\beta^{2}\right)-\varepsilon \beta^{2}(h) \rho_{2}^{(i+1)}\right]},
$$

showing (see fig.1) that $\rho_{2}^{(i)}$ is an nondecreasing function of $\rho_{2}^{(i+1)}$. Therefore, the sequence $0=\rho_{2}^{(I+1)} \geqslant \rho_{2}^{(I)} \geqslant \rho_{2}^{(I-1)} \geqslant \cdots$ is a nonincreasing nonpositive one, converging through an elementary iteration process towards the negative root $\rho_{2}^{(-\infty)}$ of

$$
\frac{\pi}{b} \varepsilon \beta^{2}(h) \rho_{2}^{2}+\left[\frac{\pi}{b}\left[\varepsilon \mathcal{F}\left(\gamma^{2}\right)-\mathcal{F}\left(\beta^{2}\right)\right]-\left[1-\varepsilon \alpha^{2}(h)\right] \mathcal{F}(1)\right] \rho_{2}-\frac{\pi}{b}\left[\mathcal{F}\left(\alpha^{2}\right) \mathcal{F}\left(\beta^{2}\right)-\mathcal{F}^{2}(\alpha \beta)\right]-\mathcal{F}(1) \mathcal{F}\left(\alpha^{2}\right)=0
$$

(c) We now return to (21) in order to show that each $(\pi / b) \rho_{2}^{(i)}(\pi / b)$ is a nonincreasing function of $\pi / b$ : indeed, it is true for $\rho_{2}^{(I+1)} \equiv 0$; if it is true for $(\pi / b) \rho_{2}^{(i+1)}(\pi / b)$, the denominator of (21) is a positive nondecreasing function, and the numerator times $\pi / b$ is a nonpositive nonincreasing function.

Therefore, from (19), $R_{i}(\pi / b)$ is a nonincreasing function of $\pi / b$ too, which must vanish for one positive value $\eta_{i}$ of $\pi / b$ (as $R_{i}(0)=\mathcal{F}(1)>0$, and $R_{i}(\infty)=-\infty$ if $i<I$ ).

Finally, as $R_{i}<R_{i+1}, \eta_{i}<\eta_{i+1}$, (see fig.2), which ends the proof of the theorem. 


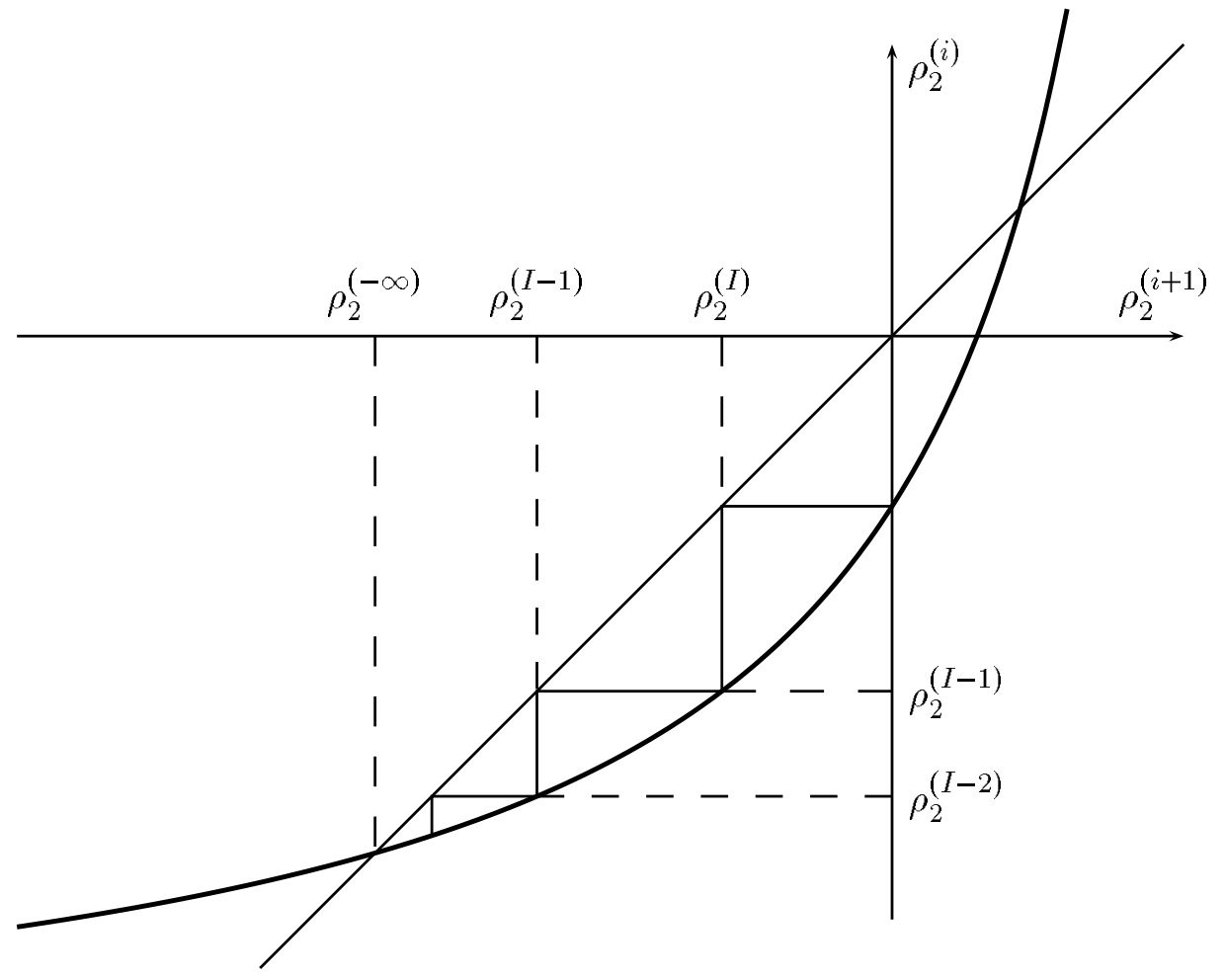

FiguRE 1. Successive values of $\rho_{2}^{(i)}$.

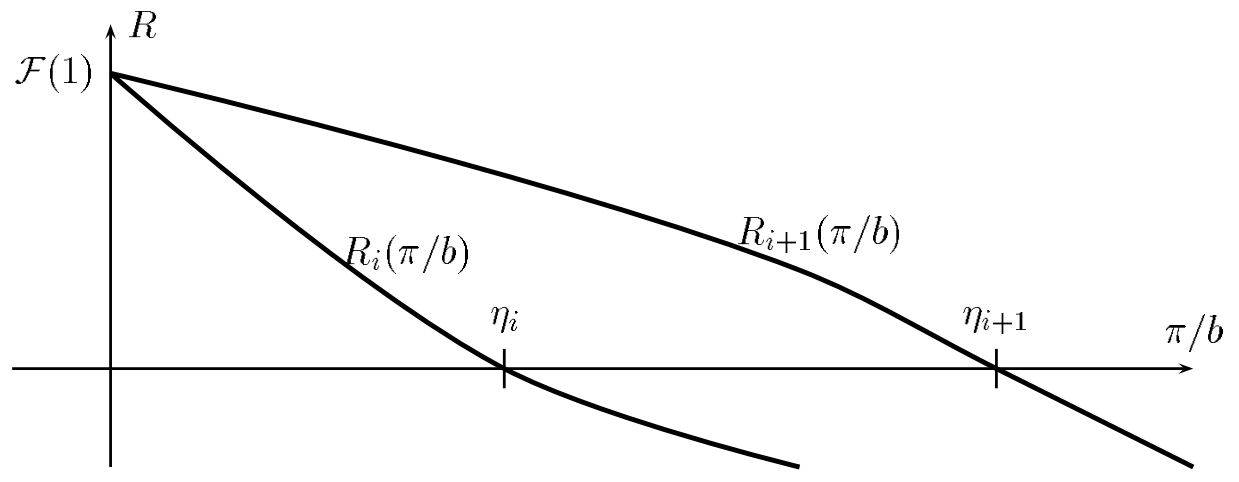

Figure 2. Zeros of two successive $R$ functions.

\section{Comparative statics.}

With the integral form of $\mathcal{F}$ from (9) above, we look at values of $\eta_{i}$, i.e., the ratio $\pi / b$ separating permits from tax, for various values of $h, \delta$, and $r$. The value $\eta^{*}$ corresponds to infinite horizon. We also show $\eta^{*}(H K)$ as calculated by Hoel and Karp [1, formula (15) p.377].

First, with $\delta=0.005, r=0.03$, and several values of $h$ :

\begin{tabular}{|c||c||c||c|c|c|c|}
\hline$h$ & $\eta^{*}(H K)$ & $\eta^{*}$ & $\eta_{I-3}$ & $\eta_{I-2}$ & $\eta_{I-1}$ & $\eta_{I}$ \\
\hline 100 & 0.00199 & 0.000839 & 0.000839 & 0.000839 & 0.000839 & 0.000991 \\
\hline 10 & 0.00897 & 0.010239 & 0.010263 & 0.010441 & 0.011857 & 0.033615 \\
\hline 1 & 0.53543 & 0.645472 & 0.655971 & 0.696356 & 0.886452 & 3.033980 \\
\hline 0.1 & 50.3504 & 61.00348 & 62.21340 & 66.55009 & 86.00395 & 300.3377 \\
\hline
\end{tabular}


With $h=10, r=0.03$, and several values of $\delta$ :

\begin{tabular}{|c||c||c||c|c|c|c|}
\hline$\delta$ & $\eta^{*}(H K)$ & $\eta^{*}$ & $\eta_{I-3}$ & $\eta_{I-2}$ & $\eta_{I-1}$ & $\eta_{I}$ \\
\hline 0.0025 & 0.00874 & 0.009690 & 0.009719 & 0.009921 & 0.011401 & 0.033004 \\
\hline 0.0050 & 0.00897 & 0.010239 & 0.010263 & 0.010441 & 0.011857 & 0.033615 \\
\hline 0.0075 & 0.00919 & 0.010796 & 0.010815 & 0.010973 & 0.012324 & 0.034234 \\
\hline 0.0100 & 0.00940 & 0.011361 & 0.011377 & 0.011516 & 0.012802 & 0.034861 \\
\hline
\end{tabular}

Finally, with $h=10, \delta=0.005$, and several values of $r$ :

\begin{tabular}{|c||c||c||c|c|c|c|}
\hline$r$ & $\eta^{*}(H K)$ & $\eta^{*}$ & $\eta_{I-3}$ & $\eta_{I-2}$ & $\eta_{I-1}$ & $\eta_{I}$ \\
\hline 0 & 0.00547 & 0.007054 & 0.007137 & 0.007495 & 0.009328 & 0.031141 \\
\hline 0.01 & 0.00652 & 0.008079 & 0.008135 & 0.008423 & 0.010119 & 0.031930 \\
\hline 0.03 & 0.00897 & 0.010239 & 0.010263 & 0.010441 & 0.011857 & 0.033615 \\
\hline 0.05 & 0.01196 & 0.012554 & 0.012564 & 0.012670 & 0.013814 & 0.035450 \\
\hline
\end{tabular}

It appears that the values of $\eta_{i}$ decrease with the length of the period $h$, and increase with the rate of depreciation $\delta$, and the rate of interest $r$. So, the lower $h$, the higher $r$ or $\delta$, the more the regulator will choose taxes. The intuition is the following: taxes are more risky in the sense that the emissions and the stock of pollutant are random (as opposed to permits). Now, damages are a quadratic function of the stock that can become very high for high shock values. But this drawback of taxes is less important when (i) the stock accumulates slowly ( $\delta$ high), (ii) the future counts less ( $r$ high), and (iii) the regulator can change rapidly of instruments ( $h$ low).

For an empirical illustration of pollution control in the framework of climate change, Hoel and Karp [1] give two estimates of $\pi / b$ which are 0.0000137 and 0.00002 . For all the values of $h, r$, and $\delta$ considered here, the tables above show that these estimates are largely lower than $\eta^{*}$, indicating that the regulator will choose the tax in all cases.

\section{References}

[1] Hoel M. and L. Karp (2002). Taxes versus quotas for a stock pollutant. Resource and Energy Economics, 24, 367-384.

[2] Hoel M. and L. Karp (2001). Taxes versus quotas for a stock pollutant with multiplicative uncertainty. Journal of Public Economics, 82, 91-114.

[3] Newell R. and W. Pizer (2003). Regulating stock externalities under uncertainty. Journal of Environmental Economics and Management, 45, 416-432.

[4] Pizer W. (2002). Combining price and quantity controls to mitigate global climate change. Journal of Public Economics, 85, 409-434. 
Département des Sciences Économiques de l'Université catholique de Louvain

Institut de Recherches Économiques et Sociales

Place Montesquieu, 3

1348 Louvain-la-Neuve, Belgique 